Effects of backlight structure on absorption experiments

C. A. Iglesias

November 12, 2004

Journal of Quantitative Spectroscopy and Radiative Transfer 
This document was prepared as an account of work sponsored by an agency of the United States Government. Neither the United States Government nor the University of California nor any of their employees, makes any warranty, express or implied, or assumes any legal liability or responsibility for the accuracy, completeness, or usefulness of any information, apparatus, product, or process disclosed, or represents that its use would not infringe privately owned rights. Reference herein to any specific commercial product, process, or service by trade name, trademark, manufacturer, or otherwise, does not necessarily constitute or imply its endorsement, recommendation, or favoring by the United States Government or the University of California. The views and opinions of authors expressed herein do not necessarily state or reflect those of the United States Government or the University of California, and shall not be used for advertising or product endorsement purposes. 


\title{
EFFECTS OF BACKLIGHT STRUCTURE ON ABSORPTION EXPERIMENTS
}

\author{
CARLOS A. IGLESIAS \\ Lawrence Livermore National Laboratory \\ P. O. Box 808, Livermore, CA 94551
}

\begin{abstract}
The impact of spectral details in the backlight of absorption spectroscopy experiments is considered. It is shown that experimentally unresolved structure in the backlight spectrum can introduce significant errors in the inferred transmission. Furthermore, it is shown that a valuable experimental procedure previously used to test the accuracy of the data fails to reveal these errors.
\end{abstract}




\section{INTRODUCTION}

The radiative properties of plasmas are of interest in a variety of fields such as astrophysics, inertial fusion, and x-ray laser production. One experimental technique to study these properties is absorption spectroscopy [1]. Such experiments have provided valuable data to test plasma absorption models. For example, large increases in stellar envelope opacities were validated [2] as well as establishing the need for line-by-line methods in low densities plasmas [3]. The technique has also been used to infer the temperature of radiatively heated samples [4].

A discussion of an experimental campaign designed to generate quantitative absorption data was given by Perry et al [5]. Although during that effort backlight sources were studied [6], details were not discussed. In fact, backlight spectra are not typically discussed or reported in these experiments (for exceptions see [1,7]). In particular, the backlight typically requires the high intensity produced by spectral lines rather continua. However, the spectral resolution is usually insufficient to determine whether the myriad lines are actually merging to form a quasicontinuum or just experimentally unresolved.

In spite of the difficulties, experiments have in general reported satisfactory agreement between data and best absorption models. Still, there remain some unexplained discrepancies $[8,9]$. If these discrepancies are due to experimental errors, it is imperative to improve the experimental techniques in future efforts. The purpose here is to consider the effect spectral details in the backlight may have on the accuracy of the extracted transmission.

\section{EXPERIMENTAL METHOD}

The absorption experiments use what in principle is a straightforward set up [5]. The procedure involves backlighting a sample material with intensity $I_{o}(v)$ where $h v$ is the photon energy. The transmission is then obtained from

$$
T(v)=\frac{I(v)}{I_{o}(v)}
$$

where $I(v)$ is the attenuated backlight signal through the material. 
In practice, however, the measurements involve an instrument function, $g(v)$, limiting the spectral resolution. Consequently, the inferred transmission is not that in Eq. (2.1) but rather

$$
T_{\text {expt }}(v)=\frac{g * I}{g * I_{O}}
$$

where the convolution is defined for arbitrary functions $g(v)$ and $f(v)$ by

$$
g * f=\int_{-\infty}^{\infty} d v^{\prime} g\left(v^{\prime}\right) f\left(v-v^{\prime}\right)
$$

For clarity, the present discussion neglects other experimental details [5], such as sample emission, gradients, and background contributions.

In contrast, theoretical models compute the transmission directly and the effects of the instrument function are simulated by

$$
T_{t h}^{g}(v)=g * T_{t h}
$$

where the theoretical transmission, $T_{t h}(v)$, is computed from the model photon absorption, $\kappa_{\text {th }}(v)$, using Beer's law [1],

$$
T_{t h}(v)=\exp \left\{-\rho L \kappa_{t h}(v)\right\}
$$

with $\rho$ and $L$ the sample mass density and thickness along the line of sight, respectively.

The agreement between experiment and theory is assessed by comparing $T_{\text {exp } t}$ and $T_{t h}^{g}$. This requires that

$$
g * T=\frac{g *\left(I_{o} T\right)}{g * I_{O}}
$$

which is not generally true. It is approximately satisfied if either $T(v)$ or $I_{o}(v)$ is a smooth function relative to the instrument resolution. However, in many cases of interest the absorption is dominated by spectral lines and the backlight often contain spectral structure. 


\section{SIMULATION OF EXPERIMENT}

The efficacy of absorption experiments depends on satisfying Eq. (2.6). In order to study this relationship, an absorption experiment is simulated. That is, the transmission and backlight spectra as well as the instrument function are generated with computer models followed by a comparison of the LHS and RHS of Eq. (2.6).

To proceed assume the instrument function is a normalized gaussian,

$$
g(v)=\frac{1}{\sigma \sqrt{\pi}} \exp \left\{-(v / \sigma)^{2}\right\}
$$

Throughout the simulation the instrument function is assigned $h \sigma=h \sigma_{g}=1 \mathrm{eV}$ representative of experiments $[1,4,5,7,8]$.

The transmission, $T_{t h}(v)$, is obtained from an aluminum $K$-shell absorption spectra [10] computed with the OPAL opacity code [11]. This Al spectrum was previously investigated and good agreement obtained between theory and experiments [4,12-14].

Typically, the backlight source consists of mid to high $\mathrm{Z}$ materials that are heated by a short, intense laser pulse; thus, they are presently impracticable to model. To simulate a backlight spectrum, the emission for Fe at stellar envelope conditions [15] was computed with the OPAL

code. The myriad spectral lines in the photon energy range near and below $100 \mathrm{eV}$ were then artificially shifted to overlap the aluminum $K$-shell spectrum.

Figure 1 displays the spectrum generated from the Fe emission as described above which was in addition convolved with a gaussian of $h \sigma=0.1 \mathrm{eV}$ to yield $I_{o}(v)$. This convolution is not associated with the instrument function but rather an arbitrary choice intended to produce a plausible backlight with spectral structure. For comparison, the figure also displays the simulated measured backlight intensity,

$$
I_{o}^{g}(v)=g * I_{O}
$$

which shows the effect of the instrument function. 
The simulated measured Al transmission with this backlight and instrument function is obtained from Eq. (2.2) and is plotted in Fig. 2 along with $T_{t h}^{g}(v)$ of Eq. (2.4). The curves show poor agreement; consequently, Eq. (2.6) is not satisfied. Note that the simulated measured backlight intensity, $I_{o}^{g}(v)$, in Fig. 1 appears sufficiently smooth to produce significantly smaller errors in the inferred transmission (less than 0.01 [16]). However, that is a false perception produced by the limited spectral resolution since the true error is significant as shown in Fig. 2. Also plotted in Fig. 2 is $T_{\text {exp } t}^{s}(v)$ produced by a shifted backlight discussed in Sect. 6.1.

\section{ELEMENTARY MODEL}

The error in Fig. 2 introduced by the unresolved spectral structure is not easily quantified. For example, $T_{\exp t}(v)$ overestimates as well as underestimates $T_{t h}^{g}(v)$. In addition, near regions of peak absorption the discrepancies can be either small or large. As argued below, the errors depend on the details of both the backlight and absorption spectra that are sufficiently complicated in the above simulation to confound analysis.

An elementary example is considered that exemplifies the main consequences of unresolved spectral details. In this example the sample transmission is defined as

$$
T_{\alpha}(v)= \begin{cases}\alpha & v_{o}-\Delta v<v<v_{o} \\ 1 & \text { otherwise }\end{cases}
$$

where $0 \leq \alpha \leq 1$ and $\Delta v$ is a fixed frequency interval. The backlight intensity, in arbitrary units, is given by

$$
I_{\mathcal{E}}(v)=\left\{\begin{array}{cl}
0 & \left|v-v_{o}\right|>\Delta v \\
1+\varepsilon & v_{o}-\gamma \Delta v \leq v \leq v_{o}+(1-\gamma) \Delta v \\
1 & \text { otherwise }
\end{array}\right.
$$

where $0 \leq \gamma \leq 1$ and $\varepsilon \geq 0$. Both $T_{\alpha}(v)$ and $I_{\varepsilon}(v)$ represent a spectral "line" added to a constant background. A schematic representation of Eqs. (4.1) and (4.2) is provided in Fig. 3. 
Finally, it is assumed that the detector does not resolve photon frequencies in the spectral range $\left|v-v_{o}\right| \leq \Delta v$. Consequently, using the backlight $I_{\varepsilon}(v)$ to measured the transmission of the sample represented by $T_{\alpha}(v)$ yields

$$
T_{\varepsilon}(\alpha)=T_{o}(\alpha)+\delta T_{\varepsilon}(\alpha)
$$

Here, $T_{o}(\alpha)$ is the result using a smooth backlight,

$$
T_{o}(\alpha)=\frac{1+\alpha}{2}
$$

The error due to unresolved spectral structure is given by

$$
\delta T_{\varepsilon}(\alpha)=(1-2 \gamma)\left(\frac{\varepsilon}{2+\varepsilon}\right)\left(\frac{1-\alpha}{2}\right)
$$

and vanishes in the limit of a smooth backlight or transmission; that is, $\varepsilon=0$ or $\alpha=1$. Although the experimental resolution is not explicit in Eq. (4.5), it is crucial to the result since it is the inability to resolve spectral details that introduces errors.

This elementary example provides insight into the problem. For example, the first bracket in the RHS of Eq. (4.5) represents the degree of "line" coincidence in the transmission and backlight. Note that for any allowed values of $\alpha$ and $\varepsilon$

$$
\begin{aligned}
& \delta T_{\varepsilon}(\alpha) \geq 0 \quad \text { for } \gamma<1 / 2 \\
& \delta T_{\varepsilon}(\alpha) \leq 0 \quad \text { for } \gamma>1 / 2
\end{aligned}
$$

with the crossing at $\gamma=1 / 2$ due to the example specifics. The second bracket describes the smoothness of the backlight spectrum. It is the ratio of the number of photons in the backlight "line" to the total number of photons in the backlight. The third bracket describes the smoothness of the transmission. Specifically, it is proportional to the difference in the number of photons transmitted through the background minus those transmitted through the "line" assuming a smooth $(\varepsilon=0)$ backlight. 
The simplicity of this example allows the factorization of the various error contributions to the "measured" transmission, which may not be possible in more complicated situations. Nevertheless, it identifies conditions that can impact absorption spectroscopy experiments.

\section{SATURATION EFFECTS}

A general feature of absorption spectroscopy is the so-called saturation of the transmission. Although this is a well-understood phenomenon, it is instructive to review the main results and observe the effects on the present examples.

The saturation occurs as a result of non-linear behavior and strongly depends on the ratio of the line widths to experimental spectral resolution $[17,18]$. The saturation effects can be experimentally observed by noting that the transmissions of two identical materials of thickness $L$ and $L^{\prime}=a L$ are related by Beer's law,

$$
T\left(v ; L^{\prime}=a L\right)=\exp \left\{-\rho L^{\prime} \kappa(v)\right\}=\exp \{-a \rho L \kappa(v)\}=T^{a}(v ; L)
$$

Experimentally Eq. (5.1) is not satisfied if the transmission is unresolved and far from unity (non-linear regime). As a result, the thinner sample typically exhibits larger photon absorption cross-section $[4,8]$.

Saturation effects for the elementary example of Sect. 4 are given in the Appendix. The saturation effects for the simulation in Sect. 3 are illustrated in Fig. 4, which displays $T_{t h}^{g}(v ; L)$. In addition, the figure displays similar calculations using half the thickness of $\mathrm{Al}$ and the result squared, $\left\{T_{t h}^{g}(v ; L / 2)\right\}^{2}$, which tests Eq. (5.1). The discrepancies are a consequence of the limited resolution. Note that in Fig. 4 there are saturation effects even for transmission values near 0.7. These are due to line-by-line OPAL calculations, which include individual spectral lines with widths in the order of a few tenths of an $\mathrm{eV}[13,14]$, that produce strong and narrow absorption features but are smeared by the assumed instrument function.

Although the presence of saturation effects in the transmission does not necessarily compromise an experiment, it does indicate that care be taken in comparing to theoretical 
models. In particular, the comparisons may require calculations by detailed line accounting models that can better reproduce the saturation effects resulting from narrow individual lines rather than statistical models that neglect such spectral details $[9,19]$.

\section{EXPERIMENTAL TESTS}

In order to increase confidence in absorption spectroscopy measurements in situations where there is limited spectral resolution, it is essential to develop alternative experimental procedures that can reveal errors in the extracted transmission. The elementary example in Sect. 4 suggests two such tests.

\subsection{Distinct backlight sources}

Equation (4.5) indicates that the error in the inferred transmission is sensitive to the details of the position and relative strength of the backlight unresolved spectral features. Thus, one possible test is to use distinct backlight sources and compare the measured transmissions $[1,20]$.

Calculations for the simulated measured transmission using Eq. (2.2) were repeated using a frequency shifted backlight intensity. That is,

$$
\begin{aligned}
& T_{\text {exp } t}^{S}(v)=\frac{g *\left(T_{t h} I_{o}^{s}\right)}{g * I_{o}^{S}} \\
& I_{o}^{S}(v)=I_{o}(v+\delta v)
\end{aligned}
$$

with $\delta v=\sigma_{g} / 2$. The results in Fig. 2 comparing $T_{t h}^{g}(v)$ to the two simulated measurements, $T_{\text {expt }}(v)$ and $T_{\text {expt }}^{S}(v)$, demonstrate the large sensitivity to unresolved spectral details in the backlight on the inferred transmission. Note that the transmission errors in Fig. 2 imply as much as a factor-of-two discrepancy in the photon absorption cross-section.

Clearly, absorption experiments based on results from both backlights $I_{o}(v)$ and $I_{o}^{S}(v)$ would identify large errors in the inferred transmission. This, as discussed in Sect. 3, would not be possible by solely relying on the measured backlight intensity, $I_{o}^{g}(v)$, in Fig. 1 . 


\subsection{Sample thickness variation}

Equation (4.5) also depends on the relative strength of the unresolved features in the transmission spectrum, which can be varied by changing the sample thickness while still keeping the photon absorption cross-section unchanged. Thus, the same backlight can be used on identical materials of different thickness. This technique has proven valuable in ascertaining the quality of data [4] (e.g., error from background subtractions, emission, gradients in space and time, etc). However, results from the elementary example in Sect. 4 (see Appendix) suggest that this procedure may not help identify errors due to unresolved spectral details. This is demonstrated with the simulation discussed in Sect. 3 .

The simulated transmission $T_{\text {expt }}(v ; L)$ is displayed in Fig. 4. Also presented is a similar

calculation, but with the sample thickness halved and the result squared, $\left\{T_{\exp t}(v ; L / 2)\right\}^{2}$, again testing Eq. (5.1). Additional analogous calculations are displayed in Fig. 4 using the shifted backlighter, $I_{o}^{S}(v)$. Although the discrepancies in these two comparisons are similar to the saturation effects exhibited by comparing $T_{t h}^{g}(v ; L)$ and $\left\{T_{t h}^{g}(v ; L / 2)\right\}^{2}$, neither shows the larger errors associated with unresolved spectral structure in Fig. 2. Therefore, agreement between measurements of identical materials of different thickness does not guarantee small errors from unresolved spectral structure.

\section{CONCLUSION}

The presence of spectral details in the backlight of absorption spectroscopy experiments was shown to be a potential source of error in the extracted transmission. That is, whenever the photon absorption and backlight spectrum contain experimentally unresolved features, the transmission measurement may be compromised. In view of these findings, it is then important for future experiments to minimize the impact on the inferred transmission due to spectral structure in the backlight. It may be difficult to accomplish this directly since the available spectral resolution may be inadequate. It is then necessary to find alternative experimental procedures to identify possible errors from unresolved backlight structure. 
One possible method is to backlight a sample with distinct sources [1,20]. Good agreement between the resulting transmissions would increase the confidence of the measurements. On the other hand, the otherwise valuable test using identical materials of different thickness fails to exhibit errors from unresolved backlight spectral structure. It is possible that such errors are responsible for unexplained discrepancies between models and experiments $[8,9]$.

Acknowledgments: It is a pleasure to recognize valuable discussions with Mark E. Foord and Richard W. Lee. Work performed under the auspices of the US Department of Energy by UC, Lawrence Livermore National Laboratory under Contract W-7405-Eng-48. 


\section{Appendix}

This Appendix uses the elementary example of Sect. 4 to examine if the transmissions from identical materials of different thickness can exhibit errors due to unresolved structure in the backlight spectrum.

To proceed, consider a material with thickness $L$ and transmission given by Eq. (4.1). Also consider a second sample of identical material but with thickness $L^{\prime}=2 L$. The choice for $L^{\prime}$ allows straightforward algebra to compute the discrepancy in Eq. (5.1). That is, using Eq. (4.3) get

$$
\Delta_{\varepsilon}(\alpha)=T_{\varepsilon}\left(\alpha^{2}\right)-T_{\varepsilon}^{2}(\alpha)=\left(\frac{1-\alpha}{2}\right)^{2}-\delta T_{\varepsilon}^{2}(\alpha)
$$

Saturation effects, independent of backlight structure, can be readily identified by considering Eq. (A.1) for a smooth $(\varepsilon=0)$ backlight. That is,

$$
\Delta_{\varepsilon=o}(\alpha)=\left(\frac{1-\alpha}{2}\right)^{2}
$$

and only vanishes for zero photon absorption $(\alpha=1)$.

Errors due to unresolved backlight structure can be isolate from saturation effects by rewriting Eq. (A.1) in terms of $\alpha$ independent quantities. That is, define $\delta T_{\varepsilon}(0)$ and $\Delta_{\varepsilon}(0)$ by

$$
\begin{aligned}
& \delta T_{\varepsilon}(\alpha)=\left(\frac{1-\alpha}{2}\right) \delta T_{\varepsilon}(0) \\
& \Delta_{\varepsilon}(\alpha)=\left(\frac{1-\alpha}{2}\right)^{2} \Delta_{\varepsilon}(0)
\end{aligned}
$$

to yield

$$
\delta T_{\varepsilon}(0)=\sqrt{1-\Delta_{\varepsilon}(0)}
$$

It follows that the discrepancy $\Delta_{\varepsilon}(\alpha)$ is not a good indicator of the error $\delta T_{\varepsilon}(\alpha)$. 


\section{REFERENCES}

[1] S.J. Davidson et al., Appl.Phys.Lett. 52, 847(1988)

[2] L. Da Silva et al, Phys.Rev.Lett. 69,4389(1992)

[3] P.T. Springer et al, JQSRT 58, 927(1997)

[4] T.S. Perry et al., Phys.Rev.Lett. 67, 3784(1991)

[5] T.S. Perry et al, Phys.Rev. E54, 5617(1996)

[6] R.W. Lee, private communication

[7] C.A. Back et al, JQSRT 58, 415(1997)

[8] T.S. Perry et al, JQSRT 54, 317(1995)

[9] C.A. Iglesias et al., JQSRT 81, 227(2003)

[10] Calculations used an $\mathrm{Al}$ mass density of $10^{-2} \mathrm{~g} / \mathrm{cm}^{3}$ and temperature of $50 \mathrm{eV}$. The $\mathrm{Al}$ areal mass density for the transmission was $2 \times 10^{-5} \mathrm{~g} / \mathrm{cm}^{2}$

[11] C.A. Iglesias \& F.J. Rogers, Ap.J. 464, 943(1996)

[12] J. Abdallah \& R.E.H. Clark, J.Appl.Phys. 69, 23(1991)

[13] C.A. Iglesias, J.K. Nash, M.H. Chen, \& F.J. Rogers, JQSRT 51, 125(1994)

[14] J. Zeng et al., Phys.Rev. E62, 7251(2000)

[15] Calculations used an Fe mass density of $10^{-4} \mathrm{~g} / \mathrm{cm}^{3}$ and temperature of $20 \mathrm{eV}$.

[16] This error is obtained by comparing $T_{t h}^{g}(v)$ with $\tilde{T}_{\text {exp } t}(v)=g *\left(T_{M} I_{o}^{g}\right) / g * I_{o}^{g}$

[17] C. Chenais-Popovics et al., Phys.Rev. A42, 4788(1990)

[18] S.J. Davidson et al, in Laser Interaction with Matter, ed. G. Velarde, E. Minguez, \& J.M. Perlado (World Scientific, Singapore, 1989)

[19] C. Chenais-Popovics et al., Phys.Rev. E65, 016413(2001)

[20] D.J. Hoarty et al, Phys.Rev.Letters (in press) 


\section{Figure CAPTIONS}

Fig. 1 - Simulated backlight intensity in arbitrary units as function of photon energy: $I_{o}(v)\left(\right.$ solid) and $I_{o}^{g}(v)$ (heavy solid).

Fig. 2 - Simulated transmission as function of photon energy: $T_{t h}^{g}(v)($ solid $), T_{\text {exp } t}(v)($ shortdash), and $T_{\text {expt }}^{s}(v)($ long-dash).

Fig. 3 - Schematic representation of Eqs. (4.1) and (4.2)

Fig. 4 - Simulated tests of Eq. (5.1) as function of photon energy:

$T_{t h}^{g}(v ; L)($ solid $)$ and $\left\{T_{t h}^{g}(v ; L / 2)\right\}^{2}($ dash $)$;

$T_{\text {exp } t}(v ; L)$ (solid) and $\left\{T_{\text {expt }}(v ; L / 2)\right\}^{2}$ (dash) shifted by 0.5 ;

$T_{\text {expt }}^{s}(v ; L)($ solid $)$ and $\left\{T_{\text {expt }}^{s}(v ; L / 2)\right\}^{2}($ dash $)$ shifted by 1.0. 


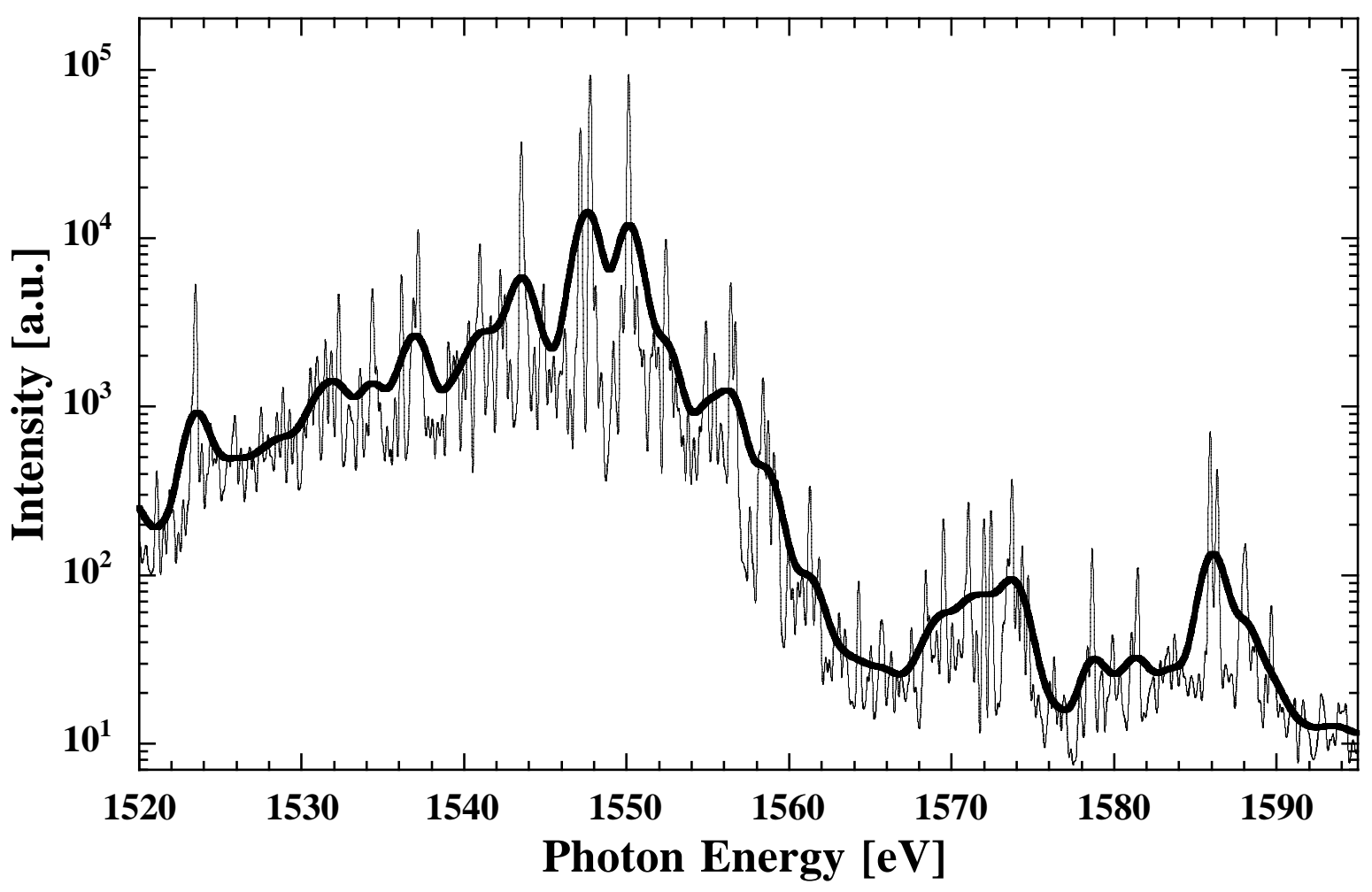

Fig. 1 


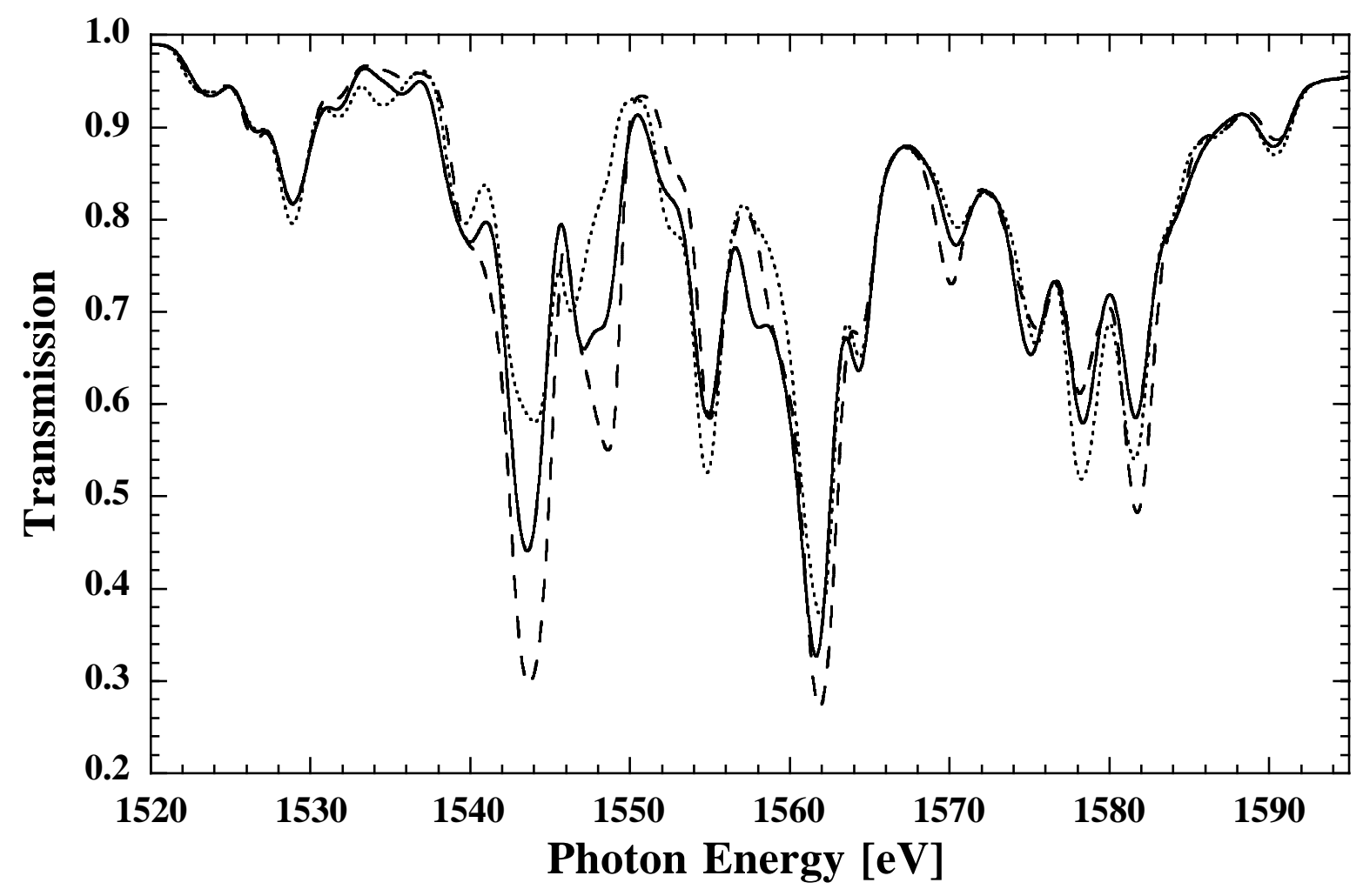

Fig. 2 


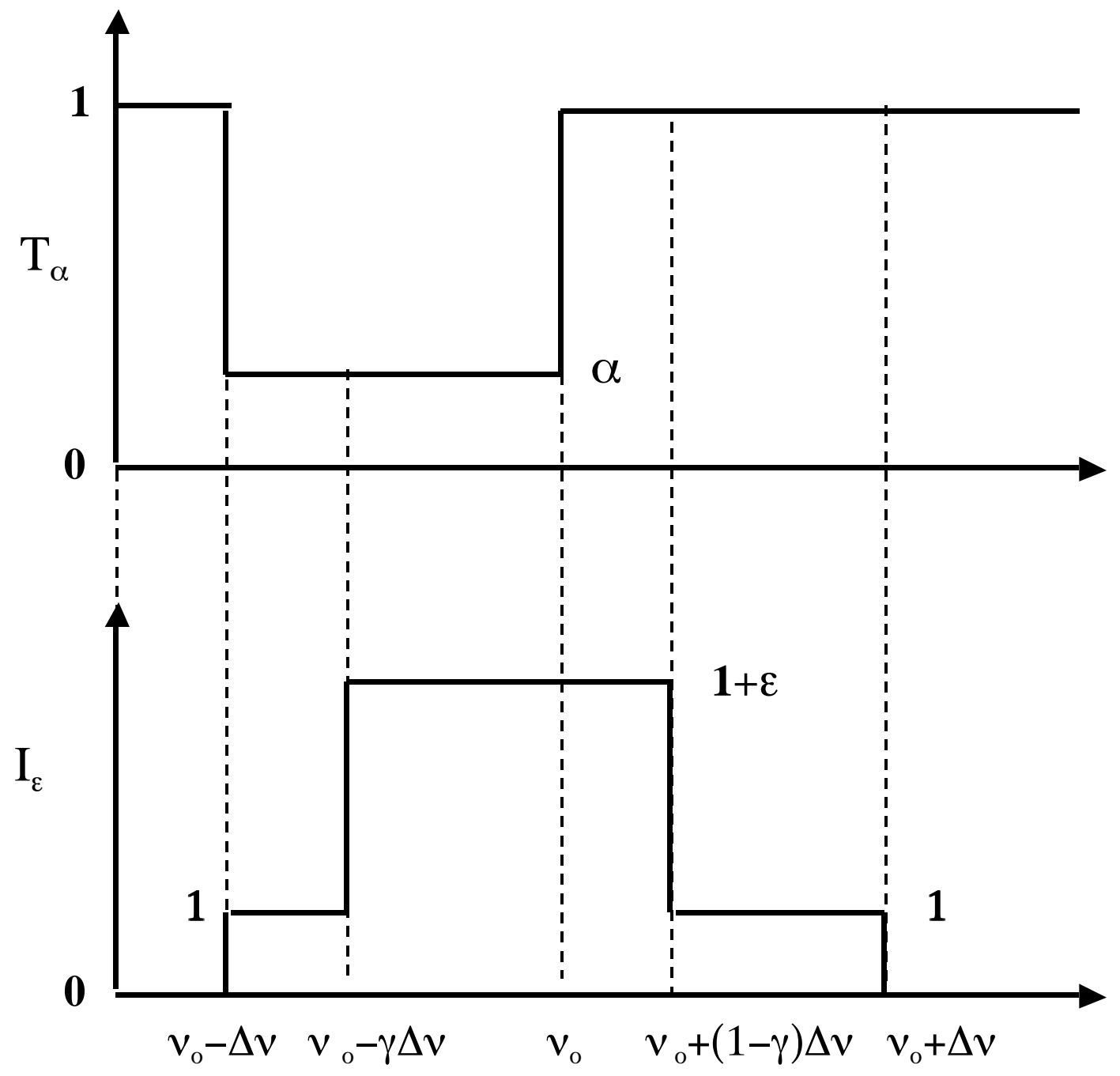

Fig. 3 


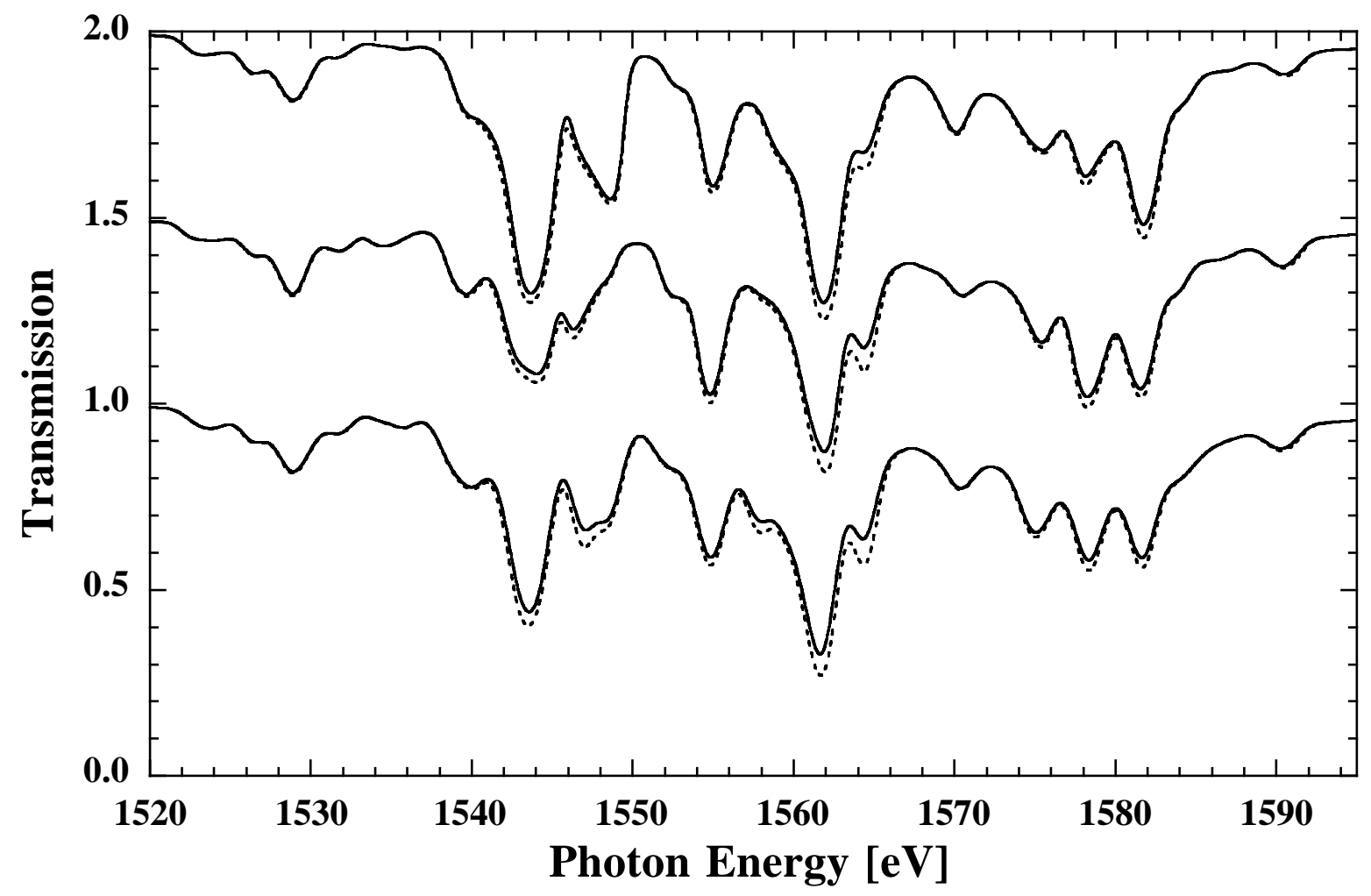

Fig. 4 